\title{
Optimality Analysis of Edge Detection Algorithms for Range Images
}

\author{
Xiaoyi Jiang \\ Department of Computer Science, University of Bern \\ Neubrückstrasse 10, CH-3012 Bern, Switzerland \\ jiang@iam.unibe.ch
}

\begin{abstract}
In this paper we propose a methodology to measure the optimality of edge detection algorithms for range images. Our optimality analysis is based on the potential of an edge detection algorithm to recover intrinsic surface information. We also propose an algorithm for actually performing the recovery. An optimality analysis on four selected edge detection algorithms demonstrate the usefulness of our approach.
\end{abstract}

\section{Introduction}

In the past years there has been an increasing interest in both theoretical and experimental evaluation of vision algorithms. In the field of range image analysis, the accuracy of curvature estimates has been studied by some researchers $[1,4,6]$. In $[7,9]$ techniques for experimental comparison of edge- and region-based range image segmentation algorithms have been proposed.

In the present paper we consider the optimality of edge detection algorithms for range images. An edge detector usually provides edge strengths that are strongly related to the local surface configuration of edge points. Thus, an edge detector generally possesses the ability of recovering intrinsic surface information (to be defined in Section 2) of edge points besides their detection. So far this surface information recovery aspect of edge detection algorithms has been entirely ignored in the literature. We define an optimal edge detector as one that allows a perfect recovery of intrinsic surface information. Measures are suggested to characterize the potential of surface information recovery of edge detection algorithms. Moreover, we propose an algorithm for actually performing this recovery.

An optimality analysis is carried out on four selected edge detection algorithms to demonstrate the usefulness of our approach by analyzing their relative performance. Such an optimality analysis can help us deepen our understanding of known algorithms. In addition, it can potentially be used to guide the design of improved edge detection algorithms. We believe that both aspects are useful to solving the important edge-based range image segmentation problem.

\section{Optimal edge detection in range images}

In range images we can distinguish between jump, crease, and smooth edges. Jump edges are relatively easy to detect. The detection of smooth edges is still 
an unsolved problem. In this paper we only consider crease edges.

The general approach to the detection of crease edges can be stated as follows. An edge detector assigns an edge strength to each pixel. Then, a thresholding operation is performed such that pixels with an edge strength higher than a specified threshold $T$ are considered as edge points.

We model the local environment of a crease edge point by two planar surfaces $z=a_{1} x+b_{1} y+c_{1}$ and $z=a_{2} x+b_{2} y+c_{2}$. Notice that since a small local environment can always be reasonably well approximated by a planar surface patch, this model is useful for curved surfaces as well. Then, the angle between the normals of the two surfaces (shortly angle of normals or AON):

$$
\cos ^{-1} \frac{\left(-a_{1},-b_{1}, 1\right) \cdot\left(-a_{2},-b_{2}, 1\right)}{\left\|\left(-a_{1},-b_{1}, 1\right)\right\| \cdot\left\|\left(-a_{2},-b_{2}, 1\right)\right\|}
$$

can be regarded as an ideal edge strength. It is independent of the position and orientation of the scene relative to the range scanner. In addition, it is invariant to changes of the coordinate system. Therefore, the AON represents an intrinsic property of edges. If it is provided by some edge detector as edge strength, the threshold $T$ needed by the thresholding operation is theoretically simply zero. In practice, however, we have to select a small angle value as threshold in order to tolerate low responses caused by noise. In [10] this discussion has motivated the definition of an optimal edge detector for range images as one that supplies the AON or a monotonic function of the AON as edge strength.

The usefulness of this definition, however, is very limited. The reason is that the majority of edge detection algorithms provides edge strengths that are not directly comparable to the AON. Moreover, the same AON can be generated by many possibilities of two intersecting planes, resulting usually in different edge strengths. In the general case, thus, an edge detector can actually be considered as a function $f(\alpha)$ that maps a value $\alpha$ of AON as defined in (1) to an interval $\left[\min _{\alpha}, \max _{\alpha}\right]$. The definition of optimal edge detectors given in [10] must be extended to treat this general case. For this purpose we require that an optimal edge detector fulfill the following two conditions:

- $\max _{\alpha}<\min _{\beta}$ for $\alpha<\beta$, i.e., the edge strength response increases with the angle of normals;

$-O_{\alpha, \beta} \equiv\left[\min _{\alpha}, \max _{\alpha}\right] \cap\left[\min _{\beta}, \max _{\beta}\right]=\emptyset$ for $\alpha \neq \beta$, i.e., the intervals corresponding to two different values of AON don't overlap.

The earlier definition of optimal edge detectors given in [10] is a special case of this general definition. The edge strength function $f(\alpha)=\alpha \equiv[\alpha, \alpha]$ obviously fulfills the two conditions above.

The following rationale lies behind the general definition of optimal edge detectors above. Although an optimal edge detector maps a value $\alpha$ of AON not to a single edge strength but to an interval, we can still distinguish edge points originating from different angles of normals. As a direct application of this fact, we can simply select $\min _{\alpha}$ as the threshold $T$ for generating a binary edge map if we want to tolerate responses caused by noise that is below the response of some 
small angle of normals $\alpha$. Moreover, we can uniquely map the edge strength of edge points back to $\mathrm{AON}$ and therefore recover the intrinsic surface information.

\section{Optimality measures}

Given an edge detection algorithm that maps a value $\alpha$ of AON to the interval $\left[\min _{\alpha}, \max _{\alpha}\right]$, we define now measures to express its optimality according to the definition of optimal edge detectors given in the last section. Without loss of generality we consider only a discrete set of AON values, say $G=\left\{0^{\circ}, 1^{\circ}, 2^{\circ}, \cdots\right\}$. For an optimal edge detector, we have $O_{\alpha, \beta}=\emptyset$ for $\alpha \neq \beta$. To which extent this condition is fulfilled can be measured by the quantity $\left|\left[\min _{\alpha}, \max _{\alpha}\right] \cap\left[\min _{\beta}, \max _{\beta}\right]\right|$ where $|[a, b]|=b-a$ means the length of the interval $[a, b]$. Generally, edge detection algorithms provide different value domains of edge strengths. In order to make them directly comparable we actually use the relative measure $\|\left[\min _{\alpha}, \max _{\alpha}\right] \cap\left[\min _{\beta}, \max _{\beta}\right]\left|/\left[\min _{\alpha}, \max _{\alpha}\right]\right|$. Considering the combination of all possible values of $\alpha$ and $\beta$, we receive the optimality measure

$$
M_{1}=\sum_{\alpha \in G, \beta \in G, \alpha \neq \beta} \frac{\|\left[\min _{\alpha}, \max _{\alpha}\right] \cap\left[\min _{\beta}, \max _{\beta}\right] \mid}{\|\left[\min _{\alpha}, \max _{\alpha}\right] \mid} .
$$

In case of optimal edge detectors this measure takes the value zero.

The optimality measure $M_{1}$ has the unwanted effect that the same amount of relative overlap contributes equally to the measure, independent of the difference between $\alpha$ and $\beta$ that produce the overlap. In general, however, we prefer small differences since otherwise it becomes difficult to distinguish between edge points caused by very different angles of normals. To take this aspect into account we add a penalty term $|\alpha-\beta|$ and receive the second optimality measure

$$
M_{2}=\sum_{\alpha \in G, \beta \in G, \alpha \neq \beta} \frac{\left.\| \min _{\alpha}, \max _{\alpha}\right] \cap\left[\min _{\beta}, \max _{\beta}\right] \mid}{\left|\left[\min _{\alpha}, \max _{\alpha}\right]\right|} \cdot|\alpha-\beta| .
$$

The optimality measures $M_{1}$ and $M_{2}$ are related to the recovery of intrinsic surface information, i.e., the angle of normals of edge points. In case of optimal edge detectors with both $M_{1}$ and $M_{2}$ being zero, the function $f(\alpha)$ is invertible and thus a perfect recovery is possible. The recovery accuracy decreases with increasing values of $M_{1}$ and $M_{2}$.

Besides surface information recovery there is another aspect that is relevant to the characterization of edge detection algorithms. Let's consider a non-optimal edge detector, and a small $\alpha$ and large $\beta$ with $O_{\alpha \beta} \neq \emptyset$. In this case we should choose the threshold $T$ smaller than the lower bound of $O_{\alpha \beta}$ in order to detect all edge points caused by the significant angle of normals $\beta$. This way we regard all edge candidates with edge strength in the range $O_{\alpha \beta}$ as true edge points. These points, however, partly correspond to the weak angle of normals $\alpha$ likely produced by noise, thus generating spurious edge points. If we set $T$ to be larger than the upper bound of $O_{\alpha \beta}$, on the other hand, we reduce the detection of spurious edge points at the risk of missing edge points produced by the significant 
angle of normals $\beta$. Generally, an overlap $O_{\alpha \beta} \neq \emptyset$ between a small $\alpha$ and a large $\beta$ indicates difficulties in the balance of reducing spurious and missing edge points. In some sense the ease of this balance is expressed in the optimality measure $M_{1}$. In order to emphasize that only situations are relevant where small values of $\mathrm{AON}$ are involved, we add a penalty term $(180-\min (\alpha, \beta))$ to $M_{1}$ and receive the third optimality measure

$$
M_{3}=\sum_{\alpha \in G, \beta \in G, \alpha \neq \beta} \frac{\left|\left[\min _{\alpha}, \max _{\alpha}\right] \cap\left[\min _{\beta}, \max _{\beta}\right]\right|}{\left.\| \min _{\alpha}, \max _{\alpha}\right] \mid} \cdot(180-\min (\alpha, \beta)) .
$$

Here 180 corresponds to the maximal possible value (in degrees) of $\alpha$ and $\beta$.

The monotony requirement of optimal edge detectors can be roughly measured as follows. For each $\alpha \in G$ we consider the center $c_{\alpha}$ of the interval $\left[\min _{\alpha}, \max _{\alpha}\right]$, i.e., $c_{\alpha}=\left(\max _{\alpha}+\min _{\alpha}\right) / 2$. We count for pairs $(\alpha, \beta), \alpha<\beta$, with $c_{\alpha} \geq c_{\beta}$. This way a fourth optimality measure

$$
L=\sum_{\alpha \in G, \beta \in G, \alpha<\beta} l_{\alpha \beta}, \quad l_{\alpha \beta}=\left\{\begin{array}{l}
0, c_{\alpha}<c_{\beta} \\
1, c_{\alpha} \geq c_{\beta}
\end{array}\right.
$$

is defined. A high value of $L$ indicates difficulties in distinguishing between edge points caused by different angles of normals.

In summary we have defined four optimality measures for edge detection algorithms. While $M_{1}$ and $M_{2}$ are related to the recovery of intrinsic surface information, $M_{3}$ is concerned with the balance of spurious and missing edge points. The monotony requirement of optimal edge detectors is measured by $L$. All the optimality measures have the property that an edge detector with the mapping function $f^{\prime}(\alpha)=a f(\alpha)+b$ is characterized by the same measures as one with the mapping function $f(\alpha)$. Our optimality measures are therefore invariant to linear transformations of the mapping function.

\section{Computation of optimality measures}

For the computation of the optimality measures of an edge detection algorithm the mapping function $f(\alpha)$ is assumed to be known. This function can be determined by simulation as follows. All possibilities of two intersecting planar surfaces at an edge point are considered. Theoretically, each of the intersecting planes can take an arbitrary orientation as long as both are visible, i.e., their slant angle (with the $z$-axis) is in the range $\left[0^{\circ}, 90^{\circ}\right.$ ). For practical reasons the plane orientation is limited such that its slant angle is smaller than $70^{\circ}$, since other orientations are rarely observed in range images.

A plane orientation corresponds to a point on the unit Gaussian sphere. In order to discretely enumerate all possible plane orientations we need a uniform tessellation of the unit sphere. In our simulation we apply a tessellation method based on the well-known geodesic dome constructions [8]. Starting with a regular icosahedron, each of its edges is divided into $f$ equal sections, where $f$ is called the frequency of the geodesic division. This results in $f^{2}$ triangles for each face 
and totally $20 f^{2}$ triangles. Then, this divided icosahedron is projected onto the unit sphere and the centers of all cells define an approximate uniform tessellation of the unit sphere. We have used a division of frequency 8 . Those orientations with a slant angle larger than $70^{\circ}$ are excluded from further consideration.

We consider all combinations of two plane orientations and compute for each such combination the (discrete) angle of normals $\alpha \in G$ and the edge strength supplied by the edge detection algorithm under study. This way we obtain for each value $\alpha \in G$ a set $S L_{\alpha}$ of possible edge strengths.

Let $A_{\alpha}$ and $S_{\alpha}$ be the average and standard deviation of the set $S L_{\alpha}$. We model the edge strengths resulting from edges of AON $\alpha$ by a normal distribution. Then, more than $95 \%$ of the responses caused by these edges lies in the range $A_{\alpha} \pm 2 S_{\alpha}$. Therefore, we define $f(\alpha)=\left[A_{\alpha}-2 S_{\alpha}, A_{\alpha}+2 S_{\alpha}\right]$.

\section{Recovery of intrinsic surface information}

As discussed in Section 2, the angle of normals is an intrinsic property of the surfaces around an edge point. Our optimality measures are mainly concerned with the potential of an edge detection algorithm to recover this intrinsic surface information. In this section we give an algorithm for actually doing the recovery.

If an edge detector is not optimal, there is no unique answer to the question which angle of normals produces a particular edge strength value. In the following we try to find out the most likely answer in a probabilistic sense.

Each AON value $\alpha \in G$ is associated with a normal distribution that is determined by simulation described in the last section and represents the probability of edge strength values being caused by edges of AON $\alpha$. For a particular edge strength value we compute its probabilities with regard to the normal distributions of all AON values out of $G$. The AON value that produces the maximal probability is considered as the most likely recovery solution.

Given the recovery algorithm above we are naturally interested in the recovery accuracy of an edge detection algorithm. For this purpose we perform a simulation, considering again all possibilities of two intersecting planar surfaces at an edge point. For each the edge strength provided by the edge detection algorithm is computed. Then, the recovered AON value is determined and the absolute difference to the ground truth is recorded. Assuming that the absolute differences obey a positive normal distribution (the negative side is meaningless in this context), the standard deviation of this distribution gives an overall characterization of the recovery accuracy.

\section{Edge detection algorithms under study}

The optimality measures and the recovery algorithm described in the previous sections are useful for all edge detection algorithms that provide quantitative edge strengths. In this work we have performed a concrete optimality analysis on four selected edge detection algorithms. A popular class of edge detection algorithms applies step edge detection operators developed in the intensity 
image domain independently to the three components of the normals of the imaged surfaces and then combine the three results. Typical examples of this class are described, for instance, in $[3,9]$ based on a morphological edge detection method and the Canny operator. Let $\left(a_{1}^{*}, b_{1}^{*}, c_{1}^{*}\right)$ and $\left(a_{2}^{*}, b_{2}^{*}, c_{2}^{*}\right)$ be the unit surface normals of the two intersecting planes of an edge point. The edge strength provided by this class of edge detection algorithms is proportional to $\max \left(a_{1}^{*}-a_{2}^{*}, b_{1}^{*}-b_{2}^{*}, c_{1}^{*}-c_{2}^{*}\right)$ that is used in our evaluation.

In [10] an edge detection method based on scan line approximation is proposed. Four directional curves (horizontal, vertical, and two diagonals) centered at a pixel on the imaged surfaces are considered. For each direction the angle between the normals of the two sides on the curve is computed. The maximal angle of the four directions gives the final edge strength.

The approach of Al-Hujazi and Sood [2] is based on residual analysis. Again, four directional curves are investigated. Both sides on a directional curve centered at a pixel are represented by a straight line and the difference between their slopes indicates the edgeness for that direction. The overall edge strength is given by the maximal slope difference of the four directions.

In [5] Zernike-moments are used to compute an edge strength that is roughly the difference between the slopes of the two intersecting planes around an edge point.

\section{Simulation results}

In the following the four algorithms used in our study will be called algorithm A-D in the order as described in the last section. For these algorithms we have computed the optimality measures and the recovery accuracy, see Table 1 . In addition, Figure 1 shows the mapping functions $f(\alpha)$. The $x$-axis represents the angle of normals $\alpha$. For each $\alpha$, the interval $\left[\min _{\alpha}, \max _{\alpha}\right]$ is drawn as a vertical line with the center (average) point marked as well. In order to make a direct comparison possible, the mapping functions have been normalized to an overall edge strength range $[0,1]$. In all algorithms the measure $L$ is very small, indicating the general monotonic trend of the edge strength. This can be easily verified by the average curves drawn in Figure 1 . The measures $M_{1}, M_{2}$ and $M_{3}$ suggest an optimality ranking Alg. B $>$ Alg. A $>$ Alg. C $>$ Alg. D. But the recovery accuracy reveals that algorithm $B$ is slightly worse than algorithm A, while the other two algorithms demonstrated a high inaccuracy. It seems that the recovery accuracy is dependent on the shape of the average curve. In general, a linear average curve indicates the potential of an accurate recovery, as exemplified by the first two algorithms in Figure 1. The mapping function of algorithm $\mathrm{D}$ is particularly interesting. The average curve is very flat in the first half of AON values. The response intervals overlap even for significantly different AON values, say $10^{\circ}$ and $70^{\circ}$. This reveals the general difficulty of this edge detection approach in balancing spurious and missing edges. Overall, our measures are able to characterize the optimality of edge detection algorithms. 


\begin{tabular}{|l||c|c|c|c|c|}
\hline & $M_{1}$ & $M_{2}$ & $M_{3}$ & $L$ & Recovery accuracy \\
\hline Alg. A & 0.22 & 2.92 & 21.73 & 91 & $8.3^{\circ}$ \\
\hline Alg. B & 0.18 & 1.82 & 20.79 & 5 & $8.9^{\circ}$ \\
\hline Alg. C & 0.28 & 4.30 & 34.41 & 19 & $14.6^{\circ}$ \\
\hline Alg. D & 0.33 & 6.04 & 41.84 & 26 & $17.4^{\circ}$ \\
\hline
\end{tabular}

Table 1. Optimality measures and recovery accuracy.
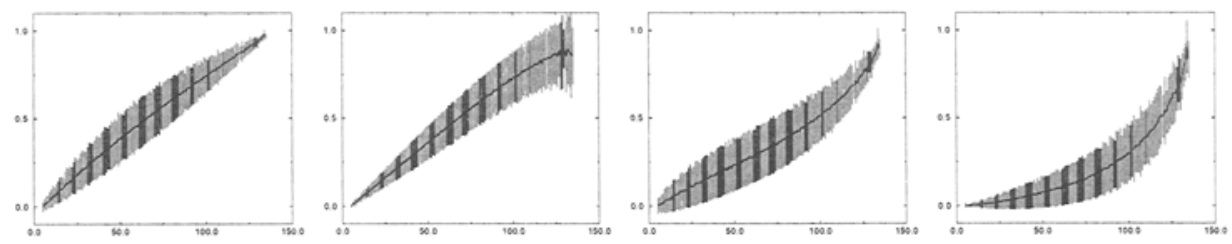

Fig. 1. From left to right the mapping functions of algorithms A-D are drawn.

Now we look at the recovery accuracy in more detail. Figure 2(a) shows the recovery accuracy as a function of AON. The behavior of algorithms $\mathrm{C}$ and $\mathrm{D}$ are quite similar. For algorithm $A$ the recovery accuracy decreases with AON so that in the area of large AON values (about $\geq 80^{\circ}$ ), algorithm $B$ becomes better. The overall recovery accuracy is certainly affected by the occurrence frequency of AON that is drawn in Figure 2(b).

There is another way to look at the recovery accuracy. The plane containing the surface normals of the two intersecting planes of an edge point makes an angle $\theta$ with the $z$-axis. Even for the same angle of normals many edge detectors produce quite different edge strengths dependent on the angle $\theta$. It is thus interesting to see the recovery accuracy as a function of $\theta$. This relationship is shown in Figure 2, together with the occurrence frequency of $\theta$. Again, algorithms $\mathrm{C}$ and $\mathrm{D}$ demonstrated a similar behavior. Interestingly, algorithm $\mathrm{B}$ is superior to algorithm $\mathrm{A}$ for $\theta$ smaller than about $35^{\circ}$. But afterwards the accuracy decreases steeply while the recovery accuracy of algorithm $A$ increases slightly.

\section{Discussions and conclusion}

In the present paper we have proposed a methodology to measure the optimality of edge detection algorithms for range images. Our optimality analysis is based on the potential of an edge detection algorithm to recover intrinsic surface information. We have also proposed an algorithm for actually performing the recovery.

The proposed optimality analysis is useful in two ways. It can help us deepen our understanding of known algorithms. The optimality measures and the detailed analysis of the simulation results enable us to study the performance of recovering intrinsic surface information of edge detection algorithms from different views, as exemplified in this work for four selected edge detection algorithms. 


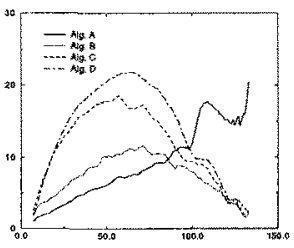

(a)

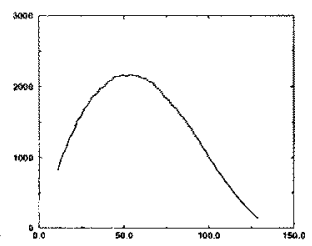

(b)

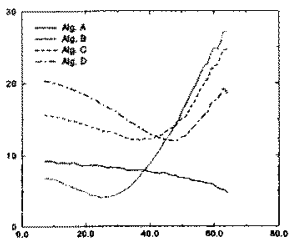

(c)

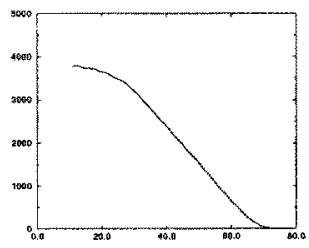

(d)

Fig. 2. Recovery accuracy as a function of $A O N(a)$ and the occurrence frequency of $A O N(b)$. Recovery accuracy as a function of $\theta$ (c) and the occurrence frequency of $\theta$ (d).

Our optimality analysis can be potentially used to design new algorithms with better performance. As an example, let's consider the algorithm B. In the original work [10] the maximal angle resulting from the four directions has been suggested. However, it turned out that we get an improved recovery accuracy of $6.3^{\circ}$ by using the second largest angle. We believe that both aspects are useful to solving the important edge-based range image segmentation problem, making the proposed approach in the present paper a valuable contribution to the increasing efforts in the characterization of vision algorithms.

\section{References}

1. N.N. Abdelmalek, Algebraic error analysis for surface curvature segmentation of 3-D range images, Pattern Recognition, 23(8): 807-817, 1990.

2. E. Al-Hujazi and A. Sood, Range image segmentation with applications to robot bin-picking using vacuum gripper, IEEE Trans. on SMC, 20(6):1313-1325, 1990.

3. P. Boulanger et al., Detection of depth and orientation discontinuities in range images using mathematical morphology, Proc. of 10th Int. Conf. on Pattern Recognition, Atlantic City, 729-732, 1990.

4. P.J. Flynn and A.K. Jain, On reliable curvature estimation, Proc. of Computer Vision and Pattern Recognition, 110-116, 1989.

5. S. Ghosal and R. Mehrotra, Detection of composite edges, IEEE Trans. on Image Processing, 3(1): 14-25, 1994.

6. A. Hilton et al., Statistics of surface curvature estimates, Pattern Recognition, 28(8): 1202-1221, 1995.

7. A. Hoover et al., An experimental comparison of range image segmentation algorithms, IEEE Transactions on PAMI, 18(7): 673-689, 1996.

8. B.K.P. Horn, Robot Vision, The MIT Press, 1987.

9. X.Y. Jiang et al., A methodology for evaluating edge detection techniques for range images, Proc. of 2nd Asian Conf. on Computer Vision, Singapore, Vol.II, 415-419, 1995.

10. X.Y. Jiang and H. Bunke, Robust and fast edge detection and description in range images, Proc. of IAPR Workshop on Machine Vision Applications, Tokyo, 538-541, 1996 . 